\title{
Efficacy and Safety of Rebamipide versus Its New Formulation, AD-203, in Patients with Erosive Gastritis: A Randomized, Double- Blind, Active Control, Noninferiority, Multicenter, Phase 3 Study
}

Gwang Ha Kim¹, Hang Lak Lee², Moon Kyung Joo ${ }^{3}$, Hong Jun Park ${ }^{4}$ Sung Woo Jung ${ }^{5}$, Ok-Jae Lee ${ }^{6}$, Hyungkil $\mathrm{Kim}^{7}$, Hoon Jai Chun ${ }^{8}$, Soo Teik Lee ${ }^{9}$, Ji Won Kim ${ }^{10}$, Han Ho Jeon ${ }^{11}$, Il-Kwun Chung ${ }^{12}$, Hyun-Soo Kim ${ }^{13}$, Dong Ho $\mathrm{Lee}^{14}$, Kyoung-Oh Kim ${ }^{15}$, Yun Jeong Lim ${ }^{16}$, Seun-Ja Park ${ }^{17}$, Soo-Jeong Cho ${ }^{18}$, Byung-Wook Kim ${ }^{19}$, Kwang Hyun $\mathrm{Ko}^{20}$, Seong Woo Jeon ${ }^{21}$, Jae Gyu Kim², In-Kyung Sung ${ }^{23}$, Tae Nyeun Kim²4, Jae Kyu Sung ${ }^{25}$, and Jong-Jae Park

${ }^{1}$ Department of Internal Medicine, Pusan National University College of Medicine, and Biomedical Research Institute, Pusan National University Hospital, Busan, ${ }^{2}$ Department of Internal Medicine, Hanyang University Hospital, ${ }^{3}$ Division of Gastroenterology, Department of Internal Medicine, Korea University Guro Hospital, Seoul, ${ }^{4}$ Department of Internal Medicine, Yonsei University Wonju College of Medicine, Wonju, ${ }^{5}$ Division of Gastroenterology, Department of Internal Medicine, Korea University Ansan Hospital, Ansan, ${ }^{6}$ Department of Internal Medicine, Gyeongsang National University College of Medicine, Jinju, ${ }^{7}$ Department of Internal Medicine, Inha University School of Medicine, Incheon, ${ }^{8}$ Division of Gastroenterology and Hepatology, Department of Internal Medicine, Institute of Gastrointestinal Medical Instrument Research, Korea University College of Medicine, Seoul, ${ }^{9}$ Department of Internal Medicine, Jeonbuk National University Hospital, Jeonju, ${ }^{10}$ Department of Internal Medicine, SMG-SNU Boramae Medical Center, Seoul National University of College of Medicine, Seoul, ${ }^{11}$ Department of Internal Medicine, National Health Insurance Service Ilsan Hospital, Goyang, ${ }^{12}$ Department of Internal Medicine, Soonchunhyang University College of Medicine, Cheonan, ${ }^{13}$ Department of Internal Medicine, Chonnam National University Hospital, Gwangju, ${ }^{14}$ Department of Internal Medicine, Seoul National University Bundang Hospital, Seoul National University College of Medicine, Seongnam, ${ }^{15}$ Department of Internal Medicine, Gachon University Gil Medical Center, Incheon, ${ }^{16}$ Department of Internal Medicine, Dongguk University College of Medicine, Seoul, ${ }^{17}$ Department of Internal Medicine, Kosin University Gospel Hospital, Kosin University College of Medicine, Busan, ${ }^{18}$ Department of Internal Medicine, Seoul National University College of Medicine, ${ }^{19}$ Department of Internal Medicine, Incheon St. Mary's Hospital, College of Medicine, The Catholic University of Korea, Seoul, ${ }^{20}$ Department of Internal Medicine, CHA Bundang Medical Center, CHA University, Seongnam, ${ }^{21}$ Department of Internal Medicine, School of Medicine, Kyungpook National University, Daegu, ${ }^{22}$ Department of Internal Medicine, Chung-Ang University College of Medicine, ${ }^{23}$ Department of Internal Medicine, Konkuk University School of Medicine, Seoul, ${ }^{24}$ Department of Internal Medicine, Yeungnam University College of Medicine, Daegu, and ${ }^{25}$ Department of Internal Medicine, Chungnam National University College of Medicine, Daejeon, Korea

Article Info

Received November 12, 2020

Revised December 10, 2020

Accepted December 28, 2020

\section{Corresponding Author}

Jong-Jae Park

ORCID https://orcid.org/0000-0002-4642-5405

E-mail gi7pjj@korea.ac.kr
Background/Aims: The mucoprotective drug rebamipide is used to treat gastritis and peptic ulcers. We compared the efficacy of Mucosta ${ }^{\circledR}$ (rebamipide $100 \mathrm{mg}$ ) and its new formulation, AD203 (rebamipide $150 \mathrm{mg}$ ), in treating erosive gastritis.

Methods: This double-blind, active control, noninferiority, multicenter, phase 3 clinical trial randomly assigned 475 patients with endoscopically proven erosive gastritis to two groups: AD-203 twice daily or Mucosta ${ }^{\circledR}$ thrice daily for 2 weeks. The intention-to-treat (ITT) analysis included 454 patients (AD-203, n=229; Mucosta $\left.{ }^{\circledR}, n=225\right)$, and the per-protocol (PP) analysis included 439 patients (AD203, $n=224$; Mucosta $\left.{ }^{\circledR}, n=215\right)$. The posttreatment assessments included the primary (erosion improvement rate) and secondary endpoints (erosion and edema cure rates; improvement rates of redness, hemorrhage, and gastrointestinal symptoms). Drug-related adverse events were evaluated.

Results: According to the ITT analysis, the erosion improvement rates (posttreatment) in AD203-treated and Mucosta ${ }^{\circledR}$-treated patients were $39.7 \%$ and $43.8 \%$, respectively. According to the PP analysis, the erosion improvement rates (posttreatment) in AD-203-treated and Mucosta ${ }^{\otimes_{-}}$ treated patients were $39.3 \%$ and $43.7 \%$, respectively. The one-sided $97.5 \%$ lower limit for the improvement rate difference between the study groups was $-4.01 \%$ (95\% confidence interval [Cl], $-13.09 \%$ to $5.06 \%)$ in the ITT analysis and $-4.44 \%(95 \% \mathrm{Cl},-13.65 \%$ to $4.78 \%)$ in the PP analysis. The groups did not significantly differ in the secondary endpoints in either analysis. Twenty-four AD-203-treated and 20 Mucosta ${ }^{\circledR}$-treated patients reported adverse events but no serious adverse drug reactions; both groups presented similar adverse event rates.

Conclusions: The new formulation of rebamipide $150 \mathrm{mg}$ (AD-203) twice daily was not inferior to rebamipide $100 \mathrm{mg}\left(\right.$ Mucosta $\left.^{\circledR}\right)$ thrice daily. Both formulations showed a similar efficacy in treating erosive gastritis. (Gut Liver 2021;15:841-850)

Key Words: Adverse drug reaction; Gastritis; Intention-to-treat analysis; Phase III clinical trial; Rebamipide

Copyright $\odot$ Gut and Liver.

(i) (8) This is an Open Access article distributed under the terms of the Creative Commons Attribution Non-Commercial License (http://creativecommons.org/licenses/by-nc/4.0) which permits unrestricted non-commercial use, distribution, and reproduction in any medium, provided the original work is properly cited. 


\section{INTRODUCTION}

Gastritis is one of the most common diseases in Korean adults. Generally, its diagnosis is based on endoscopic findings, such as erosion, edema, redness, and hemorrhage. Especially, erosion as a distinct mucosal defect is observed during acute exacerbation of acute gastritis and chronic gastritis. ${ }^{1}$ The incidence of gastritis in Korea is higher than that of other digestive diseases, and the prevalence of gastritis has been gradually increasing; ${ }^{2}$ thus, there is an increasing demand for effective gastritis therapies.

Gastritis treatment mainly involves the control of symptoms and improvement in gastric lesions, which can be achieved with drugs that suppress gastric acid secretion, modulate gastrointestinal (GI) motility, and protect the gastric mucosa. Acid suppressing agents, such as proton pump inhibitors (PPIs) and $\mathrm{H}_{2}$-receptor antagonists, are commonly used with an excellent therapeutic effect in clinical practice. Especially, PPIs are effective and relatively safe drugs. However, they have following limitations; a slow onset of action due to the mechanical limitation of prodrugs, a diminished inhibitory effect on gastric acid secretion when administered after a meal, and the difficulty in controlling nocturnal acid breakthrough. ${ }^{3}$ In addition, PPIs have several adverse effects in the stomach, such as oxyntic cell and enterochromaffin-like cell hyperplasia and the occurrence of hyperplastic and fundic gland polyps due to hypergastrinemia. ${ }^{4-6}$ Furthermore, some studies have suggested that long-term PPI use might affect the progression of atrophic gastritis and the development of gastric cancer. $^{7,8}$

To overcome these limitations, gastric mucoprotective agents are frequently used alone or in combination with acid-suppressing agents. Owing to the well-documented protective effect of rebamipide on the GI tract, the drug is one of the most commonly used mucoprotective agents for acute and chronic gastritis, as well as peptic ulcers, in the real world. ${ }^{9-11}$ Rebamipide promotes the healing of gastric mucosa injury by inducing the synthesis of prostaglandin and mucous glycoprotein, inhibiting the production of reactive oxygen radicals and inflammatory cytokines, and suppressing the activity of leukocytes. ${ }^{12}$ It effectively improves endoscopic and histological parameters, along with symptom control, in patients with chronic gastritis. ${ }^{10}$ Pharmacokinetically, rebamipide is primarily absorbed from the proximal portion of the small intestine,${ }^{13}$ and its elimination half-life in blood plasma is approximately 2 hours. ${ }^{14}$ Therefore, achieving therapeutic effects in patients requires thrice-daily administration of rebamipide. However, most acid-suppressing agents are administered once or twice per day, and low compliance to the thrice-daily administration of rebamipide, which diminishes its optimal therapeutic efficacy, has become a general concern.

Recent efforts to improve patient adherence for drug intake have resulted in the release of incrementally modified drug formulations. If a drug requiring thrice-daily administration is modified to a drug that is taken once or twice a day, it can improve the therapeutic effect by increasing patients' compliance via a reduced dosing frequency. Recently, AD-203 (rebamipide 150 mg; Addpharma Co., Ltd., Yongin, Korea), a new matrix-type sustained-release formulation of rebamipide using a low-viscosity water-soluble polymer, was developed to decrease dosing frequency from rebamipide $100 \mathrm{mg}$ thrice a day to rebamipide 150 $\mathrm{mg}$ twice a day. However, whether a twice-daily dose of AD-203 improves lesions in patients with gastritis remains unclear. Therefore, we conducted a randomized, doubleblind, active control, non-inferiority, multicenter, phase 3 clinical study to compare AD-203 (twice per day) with rebamipide (thrice per day) based on safety, as well as improvements in endoscopic findings and GI symptoms, in patients with gastritis.

\section{MATERIALS AND METHODS}

\section{Study population}

This study was a randomized, double-blind, active control, non-inferiority, multicenter, phase 3 clinical trial conducted in Korea from September 2019 to February 2020. Patients with gastritis were recruited from the following 25 medical centers: Korea University Guro Hospital (Seoul), Gachon University Gil Medical Center (Incheon), Konkuk University Medical Center (Seoul), Gyeongsang National University Hospital (Jinju), Korea University Ansan Hospital (Ansan), Korea University Anam Hospital (Seoul), Kosin University Gospel Hospital (Busan), National Health Insurance Service Ilsan Hospital (Goyang), Pusan National University Hospital (Busan), Seoul National University Bundang Hospital (Seongnam), Cha University Bundang Medical Center (Seongnam), Seoul National University Hospital (Seoul), Seoul National University Boramae Medical Center (Seoul), Soonchunhyang University Cheonan Hospital (Cheonan), Yeungnam University Medical Center (Daegu), Wonju Severance Christian Hospital (Wonju), Inha University Hospital (Incheon), Chonnam National University Hospital (Gwangju), Jeonbuk National University Hospital (Jeonju), Chung-Ang University Hospital (Seoul), Chungnam National University Hospital (Daejeon), Kyungpook National University Chilgok Hospital (Daegu), Hanyang University Medical Center (Seoul), The Catholic University of Korea Incheon St. Mary's Hospital 
(Incheon), and Dongguk University Ilsan Hospital (Goyang).

We enrolled patients aged 20 to 75 years with acute or chronic gastritis and one or more gastric erosions on baseline esophagogastroduodenoscopy (EGD). The exclusion criteria were as follows: (1) patients with a history of peptic ulcer or reflux esophagitis; (2) patients who had undergone a GI surgery, such as an operation to inhibit gastric acid secretion and an esophagogastric surgery; (3) patients with a history of GI malignancy; (4) patients who had used any $\mathrm{H}_{2}$-receptor antagonists, PPIs, gastrin receptor antagonists, anticholinergic drugs (muscarinic receptor antagonists), prokinetics, prostaglandin analogs, or gastric mucosal protective agents within 2 weeks of the investigational product administration; (5) patients who should take corticosteroids, nonsteroidal anti-inflammatory drugs (NSAIDs), aspirins, or anti-thrombotic agents during the study period; (6) women who were pregnant or lactating; (7) women of childbearing age not using contraception; (8) patients with significant impairments in the hematologic, renal, cardiac, pulmonary, hematopoietic, and endocrine systems; and (9) patients with known hypersensitivity to rebamipide. The body mass index, smoking status, alcohol consumption, concurrent diseases, and concomitant medication of each patient at baseline were recorded.

This trial was conducted following the principles of good clinical practice and the Declaration of Helsinki guidelines. The study protocol was approved by the Institutional Review Board of each of the 25 participating institutions, including the Korea University Guro Hospital (IRB number: 2019GR0347). Informed consent was obtained from all subjects at the time of enrollment. This trial was registered as a standard, randomized clinical trial (ClinicalTrials.gov: NCT04066530).

\section{Randomization}

Random allocation lists (1:1 ratio) were generated using a computer program and distributed to each institution. Subjects who participated in the clinical study underwent electrocardiography, blood tests, urinalysis, and EGD screening tests. Based on the screening test results, the eligible patients were randomly allocated to the test group (AD-203) or the control group (Mucosta ${ }^{\circledR}$; Korea Otsuka Pharmaceutical Co., Ltd., Seoul, Korea). This study was conducted in a double-blind manner.

According to the allocation, patient numbers were assigned and used by the investigators to describe the investigational products provided to the subjects by clinical trial pharmacists. Participants received either AD-203 twice per day with a placebo thrice per day or Mucosta ${ }^{\circledR}$ thrice per day with a placebo twice per day for 2 weeks. Each patient visited the treating hospital for the follow-up EGD 2 weeks after initiating the medication. Compliance was determined by the number of remaining tablets per drug type at the follow-up visit. If the drug compliance was $\geq 80 \%$, the data of the patient were included in the final outcome measurements.

\section{Study assessments}

\section{1) Efficacy}

Each patient underwent an EGD at baseline and 2 weeks after treatment initiation. Based on the EGD, gastric erosion was scored from 1 to 4 (1, no visible erosion; 2 , one or two erosions; 3 , three to five erosions; 4 , more than five erosions) (Supplementary Table 1). ${ }^{15}$ The EGD results after treatment were assessed as follows: very much improved ( 4 to 1 or 3 to 1 ), much improved ( 4 to 2 or 2 to 1 ), minimally improved (4 to 3 or 3 to 2 ), no change (same score), or worse (any increase in score). The primary efficacy endpoint was the improvement rate of erosions, defined as the percentage of patients classified as much improved or very much improved at the follow-up EGD 2 weeks after treatment initiation. Before the start of the clinical trial, the principal investigators from the participating institution discussed how to assess endoscopic erosion. To ensure a unified assessment, all EGD examinations were recorded and evaluated by the principal investigators, who reconfirmed the data if the sub-investigators conducted the EGD.

The secondary efficacy endpoints were the cure rates of erosion and edema, the improvement rates of redness and hemorrhage, and the improvement rate of GI symptoms 2 weeks after treatment initiation. Cure of erosions was defined as the disappearance of all erosions. Edema was scored from 1 to 2 , redness from 1 to 4 , and hemorrhage from 1 to 5 (Supplementary Table 1); ${ }^{16}$ the improvement of these endoscopic findings were defined as $\geq 50 \%$ reduction of the initial scores at the follow-up EGD 2 weeks after treatment initiation. The GI symptoms were self-reported and consisted of epigastric pain, dyspepsia, nausea/vomiting, reflux, abdominal distention, anorexia, heartburn, and belching. ${ }^{17}$ The severity of GI symptoms was scored from 0 to 3 : 0 , absent; 1 , no interference; 2 , minimum interference; and 3, marked interference with normal daily activities or with sleep. The frequency of GI symptoms was scored from 0 to 3 : 0 , absent; 1 , once a week; 2 , two or three times a week; and 3, more than three times a week. Symptom scores were obtained by the sum of the severity score and frequency score, with the maximum score of 48 . The improvement of GI symptoms was defined as $\geq 50 \%$ reduction of the initial GI symptom scores. ${ }^{17}$ 


\section{2) Safety}

Safety assessments included adverse events (AEs) and adverse drug reactions (ADRs), including any GI symptoms and abnormalities in the electrocardiography, laboratory findings, or vital signs. Blood samples were obtained at the end of the therapy.

\section{Sample size and statistical analysis}

We estimated the sample size to achieve a non-inferiority margin (i.e., 14\%), assuming that the efficacy rate of gastric mucosal protective agents such as rebamipide and eupatilin for gastric erosions determined by EGD was $47.7 \%$, based on previous studies. ${ }^{15,18,19}$ The erosion improvement rate of $\mathrm{AD}-203$ was considered non-inferior to that of Mucosta ${ }^{\circledR}$ (control group) if the one-sided 97.5\% (equivalent to two-sided 95\%) lower limit was greater than $-14 \%$, which was the pre-specified non-inferiority margin. ${ }^{15}$ Based on this threshold parameter, the study was designed to enroll 236 patients per group using the following conditions: a type 1 error of $2.5 \%$, statistical power of $80 \%$, the one-sided test, and a dropout rate of $15 \%$.

The patient data were subjected to three types of analysis: safety set, intention-to-treat (ITT) set, and per-protocol (PP) set. The safety analysis included all data from randomly assigned subjects who took the study drugs. The ITT set analysis included all subjects who had data of primary efficacy evaluation parameters after the treatment with the clinical trial drugs. The PP set analysis was focused on subjects from the ITT analysis with data indicating that these subjects had completed the clinical trial according to the protocol. Safety data were principally based on the safety analysis. Efficacy parameters were presented as frequency and proportion (with $95 \%$ confidence interval) in each group. Statistical analyses of these parameters were performed using the t-test or Wilcoxon rank-sum test for continuous data and the chi-square or Fisher exact test for categorical data. All statistical analyses were performed using SAS, version 9.4 (SAS Institute, Cary, NC, USA).

\section{RESULTS}

\section{Allocation of patients}

To evaluate the efficacy and safety of AD-203 and to determine whether AD-203 was non-inferior to Mucosta ${ }^{\circledR}$ in patients with gastritis, 593 patients were recruited from 25 tertiary hospitals in Korea from September 2019 to February 2020. Among those, 475 were randomly assigned to either the AD-203 ( $=238)$ or Mucosta ${ }^{\circledR}(n=237)$ group. Nine patients in the AD-203 group and 12 in the Mucosta ${ }^{\circledR}$ group were excluded from the ITT analysis because of protocol violation and missing data. Therefore, $454 \mathrm{pa}$ tients (AD-203, n=229; Mucosta ${ }^{\circledR}, \mathrm{n}=225$ ) were included in the ITT analysis set. Before performing the PP analysis, five patients in the AD-203 group and 10 in the Mucosta ${ }^{\circledR}$ group were excluded because of poor compliance, protocol violation, prohibited drug intake, and consent withdrawal. Consequently, the data for 439 patients (AD-203, $n=224$; Mucosta $^{\circledR}, \mathrm{n}=215$ ) were used in the PP analysis. Fig. 1 presents the flowchart of patient progression through the study with reasons for premature discontinuation.

\section{Demographics and clinical characteristics}

Table 1 shows the demographic and clinical characteristics of patients in the two groups. There were no differences between the two groups in terms of age, sex, height, weight, body mass index, smoking status, alcohol consumption, and GI symptom scores (Table 1). The baseline endoscopic findings (erosion, edema, redness, and hemorrhage) of the patients were also comparable between the two groups (Table 2).

\section{Compliance}

Drug compliance rates throughout the treatment period were $96.9 \%$ and $94.5 \%$ in the AD-203 and the Mucosta ${ }^{\circledR}$ groups, respectively; the drug compliance rate was significantly higher in the AD-203 group than in the Mucosta ${ }^{\circledR}$ group $(\mathrm{p}<0.0001)$. Furthermore, the proportion of patients with $\geq 80 \%$ drug compliance in the AD-203 group was higher than that in the Mucosta ${ }^{\circledR}$ group (98.7\% [226/229] vs $96.0 \%$ [216/225]); however, this did not reach statistical significance $(\mathrm{p}=0.074)$.

\section{Primary efficacy assessment}

Based on the ITT analysis, the erosion improvement rates 2 weeks after treatment initiation were 39.7\% (91/229) and 43.8\% (98/224) in the AD-203 and Mucosta ${ }^{\circledR}$ groups, respectively. The one-sided $97.5 \%$ lower limit for the improvement rate difference between the two groups was $-4.01 \%$ ( $95 \%$ confidence interval, $-13.09 \%$ to $5.06 \%$ ), which was higher than the non-inferiority margin of $-14.0 \%$. In the PP analysis, the erosion improvement rates 2 weeks after treatment initiation were $39.3 \%$ (88/224) and $43.7 \%(94 / 215)$ in the AD-203 and Mucosta ${ }^{\circledR}$ groups, respectively, and the one-sided $97.5 \%$ lower limit for the improvement rate difference between the two groups was $-4.44 \%$ ( $95 \%$ confidence interval, $-13.65 \%$ to $4.78 \%$ ), which was also higher than the non-inferiority margin of $-14.0 \%$. Based on the ITT and PP analysis results, AD-203 was not inferior to Mucosta ${ }^{\circledR}$ in improving gastric erosions (Table 3). 


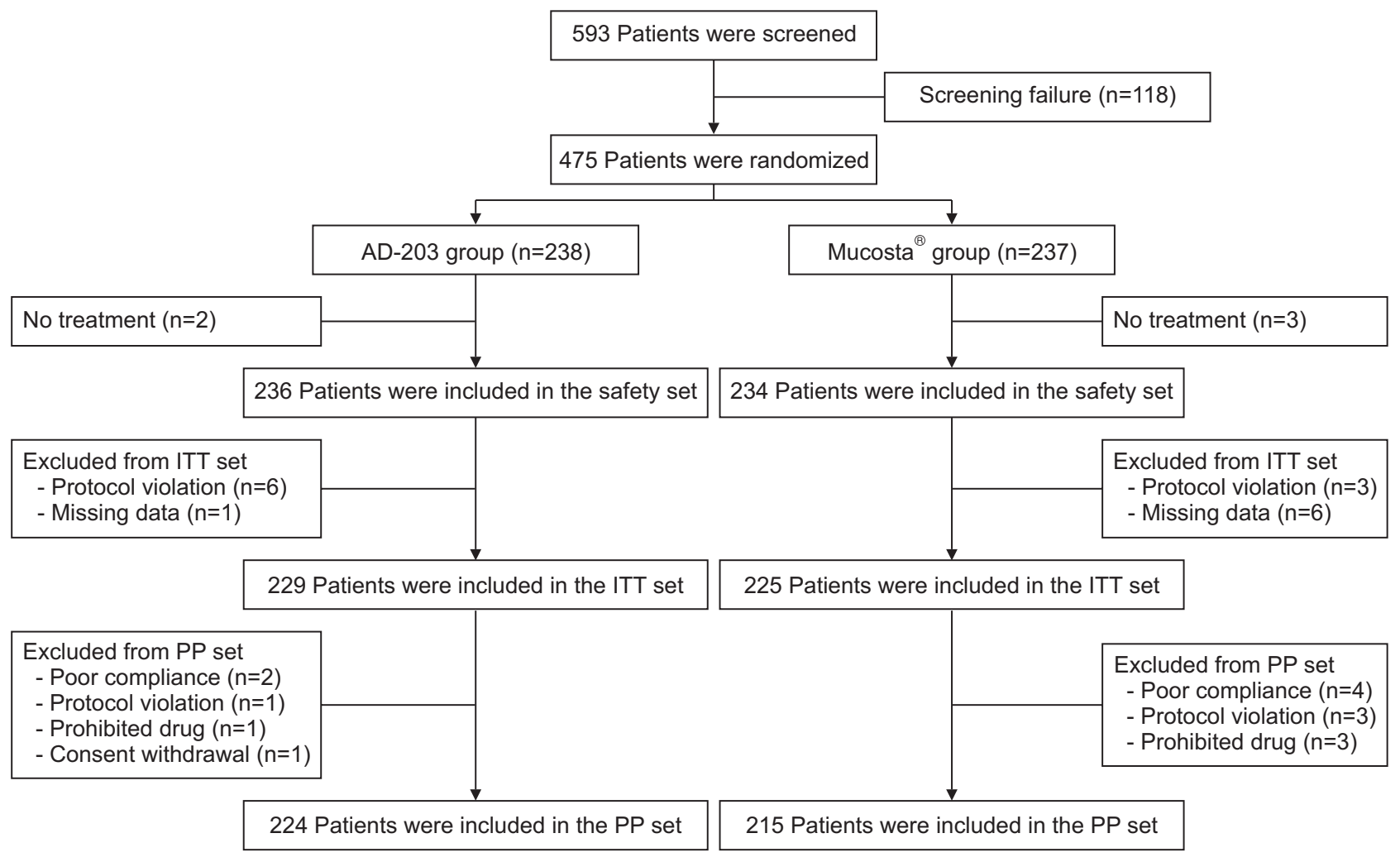

Fig. 1. Flowchart of the patients included in the study.

ITT, intention-to-treat; PP, per-protocol.

Table 1. Baseline Demographic Characteristics of the Study Patients

\begin{tabular}{|c|c|c|c|}
\hline Characteristics & AD-203 (n=229) & Mucosta $^{\circledR}(n=225)$ & $\mathrm{p}$-value \\
\hline Age, yr & $47.1 \pm 12.2$ & $46.8 \pm 12.2$ & 0.698 \\
\hline Sex & & & 0.801 \\
\hline Men & $93(40.6)$ & 94 (41.8) & \\
\hline Women & $136(59.4)$ & $131(58.2)$ & \\
\hline Height, cm & $165.2 \pm 8.7$ & $165.2 \pm 8.6$ & 0.910 \\
\hline Weight, kg & $64.4 \pm 12.0$ & $65.9 \pm 13.3$ & 0.392 \\
\hline Body mass index, $\mathrm{kg} / \mathrm{m}^{2}$ & $23.5 \pm 3.1$ & $24.0 \pm 3.3$ & 0.166 \\
\hline Smoking status & & & 0.354 \\
\hline Non-smoker & $171(74.7)$ & 174 (77.3) & \\
\hline Smoker & $43(18.8)$ & $32(14.2)$ & \\
\hline Ex-smoker & $15(6.6)$ & $19(8.4)$ & \\
\hline Alcohol consumption & & & 0.677 \\
\hline Non-drinker & $92(40.2)$ & $88(39.1)$ & \\
\hline Drinker & $132(57.6)$ & 129 (57.3) & \\
\hline Ex-drinker & 5 (2.2) & $8(3.6)$ & \\
\hline Concurrent disease & & & 0.185 \\
\hline Present & $115(50.2)$ & $99(44.0)$ & \\
\hline Absent & 114 (49.8) & $126(56.0)$ & \\
\hline Concomitant medication & & & 0.620 \\
\hline Present & 202 (88.2) & 195 (86.7) & \\
\hline Absent & $27(11.8)$ & 30 (13.3) & \\
\hline \multicolumn{4}{|c|}{ Gastrointestinal symptom scores } \\
\hline Total & $10.0 \pm 9.5$ & $9.1 \pm 9.1$ & 0.187 \\
\hline Severity & $5.1 \pm 4.9$ & $4.4 \pm 4.7$ & 0.154 \\
\hline Frequency & $5.2 \pm 4.8$ & $4.6 \pm 4.6$ & 0.181 \\
\hline
\end{tabular}

Data are presented as mean \pm SD or number (\%). 
Table 2. Baseline Endoscopic Findings in the Study Patients

\begin{tabular}{|c|c|c|c|}
\hline Endoscopic findings & $A D-203(n=229)$ & Mucosta $^{\circledast}(n=225)$ & $\mathrm{p}$-value \\
\hline Erosion & & & 0.748 \\
\hline 1 (no erosion) & 0 & 0 & \\
\hline 2 (1-2 erosions) & $82(35.8)$ & 78 (34.7) & \\
\hline 3 (3-5 erosions) & $81(35.4)$ & 75 (33.3) & \\
\hline 4 (>5 erosions) & $66(28.8)$ & 72 (32.0) & \\
\hline Edema & & & 0.214 \\
\hline 1 (none) & $118(51.5)$ & 129 (57.3) & \\
\hline 2 (pale/whiter and slightly accentuated hexagonal area gastric pattern) & $111(48.5)$ & $96(42.7)$ & \\
\hline Redness & & & 0.576 \\
\hline 1 (none) & $39(17.0)$ & $38(16.9)$ & \\
\hline 2 (minimal but obvious change) & $114(49.8)$ & $117(52.0)$ & \\
\hline 3 (conspicuous patchy discoloration) & $60(26.2)$ & $61(27.1)$ & \\
\hline 4 (color change is beefy-red in intensity) & $16(7.0)$ & $9(4.0)$ & \\
\hline Hemorrhage & & & 0.420 \\
\hline 1 (none) & $138(60.3)$ & $136(60.4)$ & \\
\hline 2 (1 hemorrhagic lesion) & $30(13.1)$ & $37(16.4)$ & \\
\hline 3 (2-5 hemorrhagic lesions) & $36(15.7)$ & $38(16.9)$ & \\
\hline 4 (6-10 hemorrhagic lesions) & 19 (8.3) & $11(4.9)$ & \\
\hline 5 (>10 hemorrhagic lesions or large area of a confluent hemorrhage) & $6(2.6)$ & $3(1.3)$ & \\
\hline
\end{tabular}

Data are presented as number (\%).

Table 3. Primary Efficacy Assessment: The Erosion Improvement Rate in the AD-203 and Mucosta ${ }^{\circledR}$ Groups

\begin{tabular}{|c|c|c|c|c|}
\hline Analysis & AD-203 & Mucosta $^{\circledast}$ & Difference $(95 \% \mathrm{CI})^{*}$ & $\mathrm{p}$-value \\
\hline \multicolumn{5}{|l|}{ Intention-to-treat set } \\
\hline No. of patients & 229 & $224^{+}$ & & \\
\hline Erosion improvement rate & $91(39.7)$ & 98 (43.8) & $-4.01(-13.09$ to 5.06$)$ & 0.387 \\
\hline \multicolumn{5}{|l|}{ Per protocol set } \\
\hline No. of patients & 224 & 215 & & \\
\hline Erosion improvement rate & 88 (39.3) & 94 (43.7) & $-4.44(-13.65$ to 4.78$)$ & 0.346 \\
\hline
\end{tabular}

Data are presented as number (\%).

$\mathrm{Cl}$, confidence interval.

*Difference is expressed as a one-sided $97.5 \%$ lower limit of the difference rate between the AD-203 and Mucosta ${ }^{\circledR}$ groups; ${ }^{\dagger}$ One patient who did not undergo follow-up endoscopy was excluded from the assessment.

\section{Secondary efficacy assessment}

The erosion cure rates determined using the ITT analysis were $34.5 \%(79 / 229)$ and $35.7 \%(80 / 224)$ in the AD203 and the Mucosta ${ }^{\circledR}$ groups, respectively (Table 4$)$. The variation in the erosion cure rate between the two groups was not statistically significant $(\mathrm{p}=0.786)$. Furthermore, the edema cure rate and the improvement rates of redness and hemorrhage did not substantially vary between the AD-203 and Mucosta ${ }^{\circledR}$ groups (31.0\% vs $29.5 \%, \mathrm{p}=0.721$; $38.9 \%$ vs $39.7 \%, p=0.850$; and $27.1 \%$ vs $28.6 \%, p=0.722$, respectively). The AD-203 and Mucosta ${ }^{\circledR}$ groups presented GI symptom improvement rates of 54.6\% (125/229) and $55.1 \%$ (124/225), respectively, which were not statistically different $(\mathrm{p}=0.910)$ (Table 5).

The erosion cure rates determined using the PP analysis were $33.9 \%(76 / 224)$ and $35.8 \%(77 / 215)$ in the $\mathrm{AD}$ 203 and the Mucosta ${ }^{\circledR}$ groups, respectively, which did not statistically differ $(\mathrm{p}=0.679)$. Furthermore, there were no differences in the cure rate of edema and the improvement rates of redness and hemorrhage between the AD-203 and Mucosta ${ }^{\circledR}$ groups $(31.3 \%$ vs $29.3 \%, \mathrm{p}=0.657 ; 38.4 \%$ vs $39.5 \%, \mathrm{p}=0.806$; and $26.8 \%$ vs $28.4 \%, \mathrm{p}=0.710$, respectively). The improvement rates of GI symptoms did not differ between the AD-203 and Mucosta ${ }^{\circledR}$ groups (54.9\% [123/224] and 55.3\% [119/215], $\mathrm{p}=0.927)$.

\section{Safety}

During the study period, 24 patients in the AD-203 group (10.2\%, 27 cases) and 20 patients in the Mucosta ${ }^{\circledR}$ group (8.5\%, 26 cases) reported AEs. Among those who reported AEs, 17 patients in the AD-203 group (7.2\%, 17 cases) and 12 patients in the Mucosta ${ }^{\circledR}$ group $(5.1 \%, 17$ cases) were confirmed to have an ADR (Table 6). GI disorders were the most common events, and the occurrence of AEs and ADRs did not significantly vary between the two groups ( $\mathrm{p}=0.546$ and $\mathrm{p}=0.350$, respectively). One patient 
Table 4. Secondary Efficacy Assessment: Analysis of Other Endoscopic Findings in the AD-203 and Mucosta ${ }^{\circledR}$ Groups

\begin{tabular}{lccc}
\multicolumn{1}{c}{ Analysis } & AD-203 & Mucosta ${ }^{\circledR}$ & p-value \\
\hline Intention-to-treat set & & & $224^{*}$ \\
No. of patients & 229 & $80(35.7)$ & 0.786 \\
Erosion cure & $79(34.5)$ & $66(29.5)$ & 0.721 \\
Edema cure & $71(31.0)$ & $89(39.7)$ & 0.850 \\
Redness improvement & $89(38.9)$ & $64(28.6)$ & 0.722 \\
Hemorrhage improvement & $62(27.1)$ & & 215 \\
Per protocol set & 224 & $77(35.8)$ & 0.679 \\
No. of patients & $76(33.9)$ & $63(29.3)$ & 0.657 \\
Erosion cure & $70(31.3)$ & $85(39.5)$ & 0.806 \\
Edema cure & $86(38.4)$ & $61(28.4)$ & 0.710 \\
Redness improvement & $60(26.8)$ & & \\
Hemorrhage improvement & & & \\
\hline
\end{tabular}

Data are presented as number (\%).

*One patient who did not undergo follow-up endoscopy was excluded from the assessment.

Table 5. Secondary Efficacy Assessment: Analysis of Gastrointestinal Symptoms in the AD-203 and Mucosta ${ }^{\circledR}$ Groups

\begin{tabular}{lccc}
\hline \multicolumn{1}{c}{ Analysis } & AD-203 & Mucosta ${ }^{\circledR}$ & p-value \\
\hline Intention-to-treat set & & & 225 \\
No. of patients & 229 & $124(55.1)$ & 0.910 \\
Total symptom improvement & $125(54.6)$ & $127(56.4)$ & 0.906 \\
$\quad$ Severity & $128(55.9)$ & $124(55.1)$ & 0.985 \\
$\quad$ Frequency & $126(55.0)$ & & 215 \\
Per protocol set & 224 & $119(55.3)$ & 0.927 \\
$\quad$ No. of patients & $123(54.9)$ & $122(56.7)$ & 0.917 \\
Total symptom improvement & $126(56.3)$ & $119(55.3)$ & 0.927 \\
$\quad$ Severity & $123(54.9)$ & & \\
$\quad$ Frequency & & & \\
\hline
\end{tabular}

Data are presented as number (\%).

in the Mucosta ${ }^{\circledR}$ group discontinued the drug due to AEs. The AE was dizziness, and its severity was mild. There were no reports of serious AEs or ADRs.

\section{DISCUSSION}

In the present study, we evaluated the efficacy and safety of AD-203, a new sustained-release rebamipide formulation (twice a day) for treating gastritis. Interestingly, AD203, as the newly developed formulation, and Mucosta ${ }^{\circledR}$, as the currently used therapeutic (thrice a day), presented similar efficacy in improving the endoscopic findings and GI symptoms in patients with gastritis. In addition, there was no difference in the reported ADRs between the AD203 and Mucosta ${ }^{\circledR}$ groups.

Rebamipide is an amino acid derivative of 2-quinolinone that enhances the defense system in the gastric mucosa via several mechanisms. In addition to inhibiting reactive oxygen species in the gastric mucosa, rebamipide stimulates prostaglandin and the prostaglandin EP4 receptor, leading to reduced gastric acid and enhanced mucus glycoprotein synthesis. ${ }^{12}$ Rebamipide also exerts anti-inflammatory effects by inhibiting superoxide anion production from neutrophils and interleukin-8 production, ${ }^{12}$ and it may influence angiogenesis, a major part of ulcer healing and tissue regeneration, that is affected by cyclooxygenase-2, vascular endothelial growth factor, nitric oxide synthase 2 , and matrix metalloproteinase- 2 . $^{20,21}$ Furthermore, rebamipide reportedly activates endothelial growth factor and its receptor expression in the gastric mucosa of rats, thereby facilitating cell proliferation and reepithelialization. ${ }^{22}$ Based on these mechanisms, rebamipide is currently used to treat gastritis, peptic ulcers, and artificial ulcers occurring after endoscopic resection for gastric epithelial neoplasms.

In a prospective study of 30 patients with chronic gastritis nonresponsive to PPIs, 8-week rebamipide treatment improved the refractory symptoms of dyspepsia, along with the endoscopic and histological features of chronic gastritis, irrespective of Helicobacter pylori infection. ${ }^{10}$ Other studies have showed that long-term rebamipide treatment improved the histological profile of gastritis and decreased the serum gastrin levels in $H$. pylori-associated 
Table 6. Incidence of Adverse Drug Reactions of the Two Medications

\begin{tabular}{|c|c|c|c|c|c|}
\hline \multirow{2}{*}{ Variable } & \multicolumn{2}{|c|}{$A D-203(n=236)$} & \multicolumn{2}{|c|}{ Mucosta $^{\circledR}(n=234)$} & \multirow{2}{*}{$\mathrm{p}$-value } \\
\hline & No. (\%) & Case & No. (\%) & Case & \\
\hline Gastrointestinal disorders & $4(1.7)$ & 4 & $3(1.3)$ & 4 & \\
\hline Nausea & $3(1.3)$ & 3 & 0 & & \\
\hline Abdominal distension & 0 & & $1(0.4)$ & 1 & \\
\hline Abdominal pain & 0 & & $1(0.4)$ & 1 & \\
\hline Constipation & 0 & & $1(0.4)$ & 1 & \\
\hline Diarrhea & 0 & & $1(0.4)$ & 1 & \\
\hline Dyspepsia & $1(0.4)$ & 1 & 0 & & \\
\hline Infections & $2(0.8)$ & 2 & $2(0.9)$ & 2 & \\
\hline Nasopharyngitis & $1(0.4)$ & 1 & $1(0.4)$ & 1 & \\
\hline Epididymitis & 0 & & $1(0.4)$ & 1 & \\
\hline Pharyngotonsillitis & $1(0.4)$ & 1 & 0 & & \\
\hline Skin disorders & $1(0.4)$ & 1 & $2(0.9)$ & 2 & \\
\hline Dry skin & 0 & & $1(0.4)$ & 1 & \\
\hline Eczema & $1(0.4)$ & 1 & 0 & & \\
\hline Pruritus & 0 & & $1(0.4)$ & 1 & \\
\hline Musculoskeletal disorders & $2(0.8)$ & 2 & $2(0.9)$ & 2 & \\
\hline Myalgia & $2(0.8)$ & 2 & $1(0.4)$ & 1 & \\
\hline Arthralgia & 0 & & $1(0.4)$ & 1 & \\
\hline Nervous system disorders & $2(0.8)$ & 2 & $2(0.9)$ & 2 & \\
\hline Dizziness & $1(0.4)$ & 1 & $1(0.4)$ & 1 & \\
\hline Headache & $1(0.4)$ & 1 & $1(0.4)$ & 1 & \\
\hline Respiratory and thoracic disorders & $2(0.8)$ & 2 & $2(0.9)$ & 2 & \\
\hline Cough & 0 & & $1(0.4)$ & 1 & \\
\hline Oropharyngeal pain & $1(0.4)$ & 1 & 0 & & \\
\hline Palpitations & 0 & & $1(0.4)$ & 1 & \\
\hline Chest discomfort & $1(0.4)$ & 1 & 0 & & \\
\hline Psychiatric disorders & $1(0.4)$ & 1 & $1(0.4)$ & 1 & \\
\hline Depression & 0 & & $1(0.4)$ & 1 & \\
\hline Insomnia & $1(0.4)$ & 1 & 0 & & \\
\hline Laboratory abnormalities & $2(0.8)$ & 2 & $1(0.4)$ & 1 & \\
\hline Leukopenia & 0 & & $1(0.4)$ & 1 & \\
\hline Blood creatine phosphokinase increase & $2(0.8)$ & 2 & 0 & & \\
\hline Others & $1(0.4)$ & 1 & $1(0.4)$ & 1 & \\
\hline Hemangioma & $1(0.4)$ & 1 & 0 & & \\
\hline Benign prostatic hyperplasia & 0 & & $1(0.4)$ & 1 & \\
\hline Total & $17(7.2)$ & 17 & $12(5.1)$ & 17 & 0.350 \\
\hline
\end{tabular}

gastritis $^{23}$ and chronic inflammation in the lesser curvature of the gastric corpus after $H$. pylori eradication. ${ }^{24}$ In a randomized, multicenter, controlled trial, the effect of rebamipide on the prevention of peptic ulcers was similar to that of misoprostol in patients on long-term NSAID therapy. ${ }^{25}$ This suggests that rebamipide is a potential therapeutic option to prevent NSAID-induced peptic ulcers.

In the present study, the overall improvement and the cure rates of gastric erosions were approximately $40 \%$ and $35 \%$, respectively. These results were similar to the efficacies of previous rebamipide and other mucoprotective agents, such as eupatilin and sulglycotide, in patients with gastritis. ${ }^{17,18}$ The improvement rate of GI symptoms in the present study was approximately $50 \%$, which is also similar to the results of previous studies on rebamipide, eupatilin, or sulglycotide use in patients with gastritis. ${ }^{9,17,18,26}$
Noncompliance is among the most commonly reported iatrogenic causes of treatment failure. Especially, the prescribed number of daily doses is inversely related to compliance. ${ }^{27}$ Failure to comply with dosage regimens can lead to suboptimal disease control, rebound symptoms, or increased readmission due to abrupt cessation. ${ }^{28,29}$ To achieve the mucoprotective efficacy of rebamipide, the drug should be taken thrice per day, but this currently prescribed medication regimen can reduce the patients' adherence to rebamipide intake. Furthermore, considering that rebamipide is usually combined with acid-suppressing agents (PPIs or $\mathrm{H}_{2}$-receptor antagonists) and NSAIDs in the treatment or prevention of gastritis and peptic ulcers, reducing the thrice-daily administration of rebamipide to that of the co-administered drugs (once or twice per day) is a critical factor for improving the adherence of patients 
to treatment. ${ }^{29}$ Thus, the sustained-release formulation of rebamipide, AD-203, was developed to mitigate the tendency to noncompliance.

The present study focused on the non-inferiority of AD-203 to Mucosta ${ }^{\circledR}$ in the treatment of gastric erosions. Both formulations significantly improved not only the endoscopic features (erosion, edema, redness, and hemorrhage) but also the GI symptoms of gastritis, and there was no difference in efficacy between AD-203 and Mucosta ${ }^{\circledR}$. Based on the safety profiles, there were no significant differences in the occurrence of AEs and ADRs between the study groups. Furthermore, although the proportion of patients with $\geq 80 \%$ drug compliance did not significantly vary between the groups, the drug compliance rate was significantly higher in the AD-203 group than in the $\mathrm{Mu}$ costa $^{\circledR}$ group. This result suggests that AD-203 could increase drug adherence, which will result in optimal disease control.

In conclusion, this study demonstrated that AD-203 administered twice daily is not inferior to Mucosta ${ }^{\circledR}$ administered thrice daily in treating gastric erosions and improving GI symptoms. With its excellent efficacy and safety profile, $\mathrm{AD}-203$ represents a promising option for treating gastritis and facilitating convenience and adherence by reducing the administration frequency.

\section{CONFLICTS OF INTEREST}

G.H.K. and B.W.K. are editorial board members of the Journal but were not involved in the peer reviewer selection, evaluation, or decision process of this article. This study was supported by Addpharma Co., Ltd. (Youngin, Korea). No other potential conflicts of interest relevant to this article were reported.

\section{AUTHOR CONTRIBUTIONS}

Analysis and interpretation of data: G.H.K., J.J.P. Drafting of the manuscript: G.H.K. Study design: J.J.P. Acquisition of data in each institute: G.H.K., H.L.L., M.K.J., H.J.P., S.W.J., O.J.L., H.K., H.J.C., S.T.L., J.W.K., H.H.J., I.K.C., H.S.K., D.H.L., K.O.K., Y.J.L., S.J.P., S.J.C., B.W.K., K.H.K., S.W.J., J.G.K., I.K.S., T.N.K., J.K.S. All authors read and approved the final manuscript.

\section{ORCID}

Gwang Ha Kim https://orcid.org/0000-0001-9721-5734
Hang Lak Lee

https://orcid.org/0000-0002-2825-3216

Moon Kyung Joo

Hong Jun Park

https://orcid.org/0000-0001-6050-3695

Sung Woo Jung

Ok-Jae Lee

https://orcid.org/0000-0001-9320-9978

https://orcid.org/0000-0003-1954-1678

https://orcid.org/0000-0002-4016-4443

Hyungkil Kim

Hoon Jai Chun

Soo Teik Lee

Ji Won Kim

Han Ho Jeon

Il-Kwun Chung

Hyun-Soo Kim

Dong Ho Lee

Kyoung-Oh Kim

Yun Jeong Lim

Seun-Ja Park

Soo-Jeong Cho

https://orcid.org/0000-0003-4230-3897

https://orcid.org/0000-0002-5539-361X

https://orcid.org/0000-0002-0758-1923

https://orcid.org/0000-0002-1214-5544

https://orcid.org/0000-0002-3393-3304

https://orcid.org/0000-0002-4158-3666

https://orcid.org/0000-0003-4834-0496

https://orcid.org/0000-0002-6376-410X

https://orcid.org/0000-0002-5365-2550

https://orcid.org/0000-0002-3279-332X

https://orcid.org/0000-0003-3217-5115

https://orcid.org/0000-0001-7144-0589

Byung-Wook Kim https://orcid.org/0000-0002-2290-4954

Kwang Hyun Ko https://orcid.org/0000-0001-5168-1377

Seong Woo Jeon https://orcid.org/0000-0002-9539-9389

Jae Gyu Kim

In-Kyung Sung

https://orcid.org/0000-0002-4841-9404

Tae Nyeun Kim

Jae Kyu Sung

Jong-Jae Park

https://orcid.org/0000-0003-4178-2056

https://orcid.org/0000-0002-9068-624X

https://orcid.org/0000-0002-4642-5405

\section{REFERENCES}

1. Laine L, Cohen H, Sloane R, Marin-Sorensen M, Weinstein WM. Interobserver agreement and predictive value of endoscopic findings for $\mathrm{H}$. pylori and gastritis in normal volunteers. Gastrointest Endosc 1995;42:420-423.

2. Park HK, Kim N, Lee SW, et al. The distribution of endoscopic gastritis in 25,536 heath check-up subjects in Korea. Korean J Helicobacter Up Gastrointest Res 2012;12:237-243.

3. Kinoshita Y, Ishimura N, Ishihara S. Advantages and disadvantages of long-term proton pump inhibitor use. J Neurogastroenterol Motil 2018;24:182-196.

4. Jalving M, Koornstra JJ, Wesseling J, Boezen HM, DE Jong S, Kleibeuker JH. Increased risk of fundic gland polyps during long-term proton pump inhibitor therapy. Aliment Pharmacol Ther 2006;24:1341-1348.

5. Raghunath AS, O'Morain C, McLoughlin RC. Review article: the long-term use of proton-pump inhibitors. Aliment Pharmacol Ther 2005;22 Suppl 1:55-63.

6. Kim GH. Proton pump inhibitor-related gastric mucosal changes. Gut Liver 2021;15:646-652.

7. García Rodríguez LA, Lagergren J, Lindblad M. Gastric acid suppression and risk of oesophageal and gastric adenocarcinoma: a nested case control study in the UK. Gut 
2006;55:1538-1544.

8. Waldum HL, Sørdal $\varnothing$, Fossmark R. Proton pump inhibitors (PPIs) may cause gastric cancer: clinical consequences. Scand J Gastroenterol 2018;53:639-642.

9. Du Y, Li Z, Zhan X, et al. Anti-inflammatory effects of rebamipide according to Helicobacter pylori status in patients with chronic erosive gastritis: a randomized sucralfatecontrolled multicenter trial in China-STARS study. Dig Dis Sci 2008;53:2886-2895.

10. Chitapanarux T, Praisontarangkul OA, Lertprasertsuke N. An open-labeled study of rebamipide treatment in chronic gastritis patients with dyspeptic symptoms refractory to proton pump inhibitors. Dig Dis Sci 2008;53:2896-2903.

11. Han X, Jiang K, Wang B, Zhou L, Chen X, Li S. Effect of rebamipide on the premalignant progression of chronic gastritis: a randomized controlled study. Clin Drug Investig 2015;35:665-673.

12. Arakawa T, Higuchi K, Fujiwara $Y$, et al. 15th anniversary of rebamipide: looking ahead to the new mechanisms and new applications. Dig Dis Sci 2005;50 Suppl 1:S3-S11.

13. Naito Y, Yoshikawa T. Rebamipide: a gastrointestinal protective drug with pleiotropic activities. Expert Rev Gastroenterol Hepatol 2010;4:261-270.

14. Hasegawa S, Sekino H, Matsuoka O, et al. Bioequivalence of rebamipide granules and tablets in healthy adult male volunteers. Clin Drug Investig 2003;23:771-779.

15. Seol SY, Kim MH, Ryu JS, Choi MG, Shin DW, Ahn BO. DA9601 for erosive gastritis: results of a double-blind placebocontrolled phase III clinical trial. World J Gastroenterol 2004;10:2379-2382.

16. Shim KN, Kim JI, Kim N, et al. The efficacy and safety of irsogladine maleate in nonsteroidal anti-inflammatory drug or aspirin-induced peptic ulcer and gastritis. Korean J Intern Med 2019;34:1008-1021.

17. Jeong JJ, Choi MG, Choi H, et al. Single blinded, randomized, active drug comparative, multi-center study to evaluate the therapeutic efficacy of gliptide ${ }^{\circledR}$ tab (sulglycotide 200 $\mathrm{mg}$ ) in gastritis patients; phase IV study. Korean J Gastrointest Endosc 2007;35:125-132.

18. Choi YJ, Lee DH, Choi MG, et al. Evaluation of the efficacy and safety of DA-9601 versus its new formulation, DA-5204, in patients with gastritis: phase III, randomized, doubleblind, non-inferiority study. J Korean Med Sci 2017;32:1807-
1813.

19. Han HW, Choi MG, Seol SY, et al. Efficacy and safety of Albis ${ }^{\circledR}$ in acute and chronic patients with gastritis: a doubleblind, placebo-controlled, randomized multi-center study. Korean J Gastrointest Endosc 2011;42:215-221.

20. Syam AF, Sadikin M, Wanandi SI, Rani AA. Molecular mechanism on healing process of peptic ulcer. Acta Med Indones 2009;41:95-98.

21. Risau W. Mechanisms of angiogenesis. Nature 1997;386:671674.

22. Tarnawski A, Arakawa T, Kobayashi K. Rebamipide treatment activates epidermal growth factor and its receptor expression in normal and ulcerated gastric mucosa in rats: one mechanism for its ulcer healing action? Dig Dis Sci 1998;43(9 Suppl):90S-98S.

23. Haruma K, Ito M, Kido S, et al. Long-term rebamipide therapy improves Helicobacter pylori-associated chronic gastritis. Dig Dis Sci 2002;47:862-867.

24. Kamada T, Sato M, Tokutomi T, et al. Rebamipide improves chronic inflammation in the lesser curvature of the corpus after Helicobacter pylori eradication: a multicenter study. Biomed Res Int 2015;2015:865146.

25. Kim HK, Kim JI, Kim JK, et al. Preventive effects of rebamipide on NSAID-induced gastric mucosal injury and reduction of gastric mucosal blood flow in healthy volunteers. Dig Dis Sci 2007;52:1776-1782.

26. Miwa H, Osada T, Nagahara A, et al. Effect of a gastroprotective agent, rebamipide, on symptom improvement in patients with functional dyspepsia: a double-blind placebo-controlled study in Japan. J Gastroenterol Hepatol 2006;21:1826-1831.

27. Claxton AJ, Cramer J, Pierce C. A systematic review of the associations between dose regimens and medication compliance. Clin Ther 2001;23:1296-1310.

28. Garfinkel D, Mangin D. Feasibility study of a systematic approach for discontinuation of multiple medications in older adults: addressing polypharmacy. Arch Intern Med 2010;170:1648-1654.

29. Toh MR, Teo V, Kwan YH, Raaj S, Tan SY, Tan JZ. Association between number of doses per day, number of medications and patient's non-compliance, and frequency of readmissions in a multi-ethnic Asian population. Prev Med Rep 2014;1:43-47. 